\title{
La seguridad del paciente en urgencias y emergencias
}

\section{Patient safety in emergency care}

\author{
S. Tomás ${ }^{1}$, I. Gimena ${ }^{2}$
}

\section{RESUMEN}

La seguridad del paciente es una dimensión de la calidad imprescindible en los servicios de urgencias (SU). La incidencia de aparición de eventos adversos (EA) en dichos servicios se estima entre un 1,6 y un $14 \%$ según diferentes estudios y metodologías. Las estrategias para conseguir una práctica clínica segura en los SU pasan por políticas dirigidas a mejorar la seguridad en el empleo de la medicación con especial énfasis en la administración y en la conciliación de la medicación, así como en el empleo de fármacos de alto riesgo; en prevenir la infección nosocomial debida a la atención urgente, con especial importancia a la higiene de manos; en mejorar la identificación de pacientes, en la mejora de la comunicación e información, especialmente durante las transferencias del paciente, en la prevención de riesgos específicos y sobre todo, en crear un clima y una cultura en seguridad del paciente en el SU.

Se disponen de diferentes herramientas proactivas y reactivas para valorar la seguridad del paciente en los SU y destaca la necesidad de promulgar los sistemas de notificación de eventos para facilitar su análisis y el posterior desarrollo de acciones de mejora en los SU.

Palabras clave. Urgencias. Seguridad Paciente. EVADUR. Eventos adversos. Gestión de riesgos.

\begin{abstract}
Patient safety is an essential dimension of quality in Emergency Departments (EDs). The incidence of appearance of adverse events in these departments is estimated at between 1.6 and $14 \%$ according to different studies and methodologies. The strategies for achieving a safe clinical practice in EDs involve policies aimed at improving safety in the use of medication with special emphasis on the administration and on the reconciliation of the medication, as well as on the employment of high risk drugs; on preventing nosocomial infection due to emergency care, with special importance manual hygiene; on improving patient identification; on improving communication and information, especially during patient transfers; on the prevention of specific risks; and, above all, on creating a climate and culture of patient safety in EDs.

Different proactive and reactive tools are available for evaluating patient safety in EDs. There is an outstanding need to publish the system of event notification to facilitate analysis of such events and the subsequent development of improvement actions in EDs.
\end{abstract}

Key words. Emergency care. Patient safety. EVADUR study. Adverse events. Risk management.
1. Servicio de Urgencias. Hospital Municipal de Badalona. Serveis Assistencials.

2. Servicio de Urgencias. Hospital de Navarra. Pamplona.

\author{
Correspondencia \\ Dr. Santiago Tomás Vecina \\ Badalona Serveis Assistencials \\ Vía Augusta, 9-13 \\ 08911 Badalona \\ E-mail: stomas@bsa.cat
}




\section{INTRODUCCIÓN}

El estudio de los efectos adversos (EA) debidos a la asistencia sanitaria ha cobrado un notable auge en los últimos años ya que son un problema frecuente, tanto por la mayor complejidad de la práctica clínica, como por el aumento de la edad media de los pacientes que son atendidos en los hospitales.

Los servicios de urgencia (SU) son, en la mayoría de las ocasiones, la puerta de entrada al sistema sanitario. Constituyen, a la vez, un servicio receptor de pacientes y un servicio proveedor de los mismos a otros niveles asistenciales, ya que desde los SU, los pacientes son ingresados, deri- vados a atención primaria o a atención especializada.

Aunque muchos de los estudios realizados sobre EA se han llevado a cabo sobre la hospitalización, éstos pueden ocurrir en cualquier escenario y, los SU, por su especial idiosincrasia, no se escapan a ellos. Los diferentes modelos organizativos junto con las caracteristicas intrínsecas de la atención urgente (Tabla 1) pueden facilitar el riesgo de aparición de EA ${ }^{1}$. Además, algunos trabajos han destacado el grado de «inadecuación" de las admisiones, estancias y/o visitas urgentes como un fenómeno a mejorar para evitar riesgos así como consumos sanitarios innecesarios ${ }^{2,3}$.

Tabla 1. Algunos factores favorecedores de errores en urgencias.

\begin{tabular}{|c|}
\hline Tipo de paciente y gravedad \\
\hline Profesionales: experiencia \\
\hline $\begin{array}{l}\text { - Médicos: } \\
\text { - formación heterogénea } \\
\text { - Plantilla, guardias, personal eventual, MIR... } \\
\text { - Falta de seguimiento del paciente } \\
\text { - Enfermería } \\
\text { - Motivación (burn out) }\end{array}$ \\
\hline Error en comunicación: \\
\hline $\begin{array}{l}\text { - Profesional - paciente } \\
\text { - Profesional - profesional } \\
\text { - Cambios turno o guardia } \\
\text { - Errores medicación: } \\
\text { - Órdenes verbales } \\
\text { - Mala letra: órdenes médicas, recetas } \\
\text { - No revisión por Farmacia }\end{array}$ \\
\hline Condiciones de trabajo: \\
\hline $\begin{array}{l}\text { - Escasa información sobre el paciente } \\
\text { - Paciente desconocido } \\
\text { - Dificultad acceso a historia clínica } \\
\text { - Interrupciones y distracciones } \\
\text { - Turnos de trabajo: } \\
\text { - Guardias: cansancio, interrupción del sueño } \\
\text { - Turnos: intercambios de información; mayor nº intervenciones por paciente } \\
\text { - Presión asistencial } \\
\text { - Ratio médico-enfermera / paciente } \\
\text { - Ubicación inadecuada } \\
\text { - Cambio ubicación del enfermo } \\
\text { - Falta de trabajo en equipo }\end{array}$ \\
\hline
\end{tabular}

Adaptado de Campodarve I. Errors Mèdics als Serveis d’urgències. ANNALS DE MEDICINA 2002; 85 (3). 
La incidencia y/o prevalencia de EA en los SU no está determinada. La mayoría de los estudios se han realizado sobre pacientes hospitalizados que fueron atendidos previamente en servicios de urgencias, pero, si tenemos en cuenta que sólo entre el 10 y el $15 \%$ de los pacientes que acuden a estos servicios son finamente hospitalizados, desconocemos la ocurrencia de efectos adversos en el alto porcentaje de pacientes que no son ingresados, que son la gran mayoría de las atenciones urgentes que se practican. Tampoco se conoce el peso que suponen las situaciones clínicas latentes que pueden acabar desencadenando un EA y cuya detección podría ayudar a prevenir la aparición del mismo. La detección y prevención del riesgo de aparición de un EA, antes de que suceda, ha de ser un objetivo primordial en la mejora de la calidad de todo SU. Por otro lado, se estima que la gran mayoría de los EA no se declaran, a pesar de la existencia de sistemas de notificación voluntaria anónima en muchos centros sanitarios. Además, probablemente los SU pueden ser un escenario adecuado para identificar efectos adversos generados en la atención primaria que provocan una visita urgente hospitalaria.

Los SU han ido introduciendo progresivamente herramientas de trabajo y elementos de gestión que han contribuido, de manera directa o indirecta, a la mejora de la seguridad del paciente. La implantación de los sistemas de triaje estructurado en los $\mathrm{SU}^{4}$, en los que se valora la priorización de la visita junto con la gravedad estimada por protocolos validados, y las políticas de mejora de la calidad mediante el seguimiento de indicadores y/o modelos de acreditación de $\mathrm{SU}^{5}$, donde se valoran, entre otros elementos, la seguridad y el riesgo, han sido algunos de los avances más significativos, promovidos especialmente desde las sociedades científicas de medicina de urgencias. En los últimos años, y gracias al fuerte empuje provocado por las alianzas en materias de la seguridad del paciente desarrolladas por las administraciones, se está implantando paulatinamente una conciencia y cultura de la seguridad entre los profesionales de la atención urgente así como el desarrollo de iniciativas de investigación con la consiguiente mejora de resultados y eficiencia en la prevención de riesgos $^{6}$.

\section{INCIDENCIA Y PREVALENCIA DE EVENTOS ADVERSOS EN LOS SERVICIOS DE URGENCIAS}

Es difícil establecer una estimación sobre la incidencia y/o prevalencia de EA en los SU, lo más aproximada a la realidad de estos servicios y de la atención urgente, debido a la diversidad metodológica de los diferentes estudios sobre EA en hospitales y en urgencias.

La mayoría de estos estudios suelen ser retrospectivos y basados en la revisión de historias, hecho que da lugar al fenómeno de "la punta de iceberg", es decir, a una detección inferior a la que pueda acontecer en realidad (todo lo que no esté escrito o no conste en la historia no se detecta). A su vez, suelen estar basados en pacientes hospitalizados que pasaron previamente por urgencias y se omiten la gran mayoría de pacientes que son dados de alta a su domicilio (entre el 80 y el $90 \%$ de los pacientes en nuestro medio), y por tanto, la posible aparición de EA tardíos, fuera del ámbito hospitalario.

En este sentido, Forster y $\mathrm{col}^{7}$ encontraron, tras revisar 399 historias, una prevalencia de EA en el 6\% de las visitas urgentes, mientras que en el metanálisis publicado por De Vries y colaboradores ${ }^{8}$, el $80 \%$ de los EA detectados acontecieron durante la hospitalización y, entre ellos, un $3 \%$ se generaron en el SU.

¿Cuál es la situación en los SU españoles? En nuestro medio hay una escasez de estudios diseñados y realizados específicamente en y para SU. La explicación al fenómeno puede buscarse en el hecho de que la preocupación por la seguridad, a pesar de su larga existencia, es relativamente joven en nuestro ámbito asistencial, teniendo, por ello, que recurrir al análisis de los resultados obtenidos en estudios globales practicados sobre la hospitalización para poder obtener una aproximación sobre lo que ocurre en la atención urgente. 
En este sentido queremos resaltar los datos que aportan dos iniciativas estatales, los estudios ENEAS y APEAS, así como otra individual, el estudio EVADUR, para hacernos una idea sobre dónde están en los SU españoles.

\section{Estudio ENEAS 9}

Este estudio fue realizado en el año 2005 y analiza un total de 24 hospitales. Se recogen un total de 665 eventos adversos (Tabla 2). Un 9,3\% de EA están relacionados con la asistencia sanitaria (un 8,4\% con la asistencia hospitalaria directa), y se consideran como leves un $45 \%$ de los mismos, moderados un $39 \%$ y graves un $16 \%$. Se constata una tasa de 1,4 EA por cada 100 días de estancia hospitalaria del paciente. Un 25\% de los EA que acontecen en los hospitales tienen que ver con procedimientos realizados (muchos de ellos realizados en urgencias), de los que podrían evitarse un $31,7 \%$, al igual que el $34,9 \%$ de los relacionados con medicación. En total un $42,8 \%$ de los EA se consideraron evitables.

A raíz de los datos de este estudio, Requena y Aranaz ${ }^{10}$ extrajeron aquella serie de datos que estaban asociados a la asistencia sanitaria en los SU y determinaron, a raíz de los mismos, la proporción de EA relacionados con la asistencia en el área de urgencias, las causas inmediatas y los impactos en cuanto a gravedad, prolongación de estancia y posible evitabilidad. Los autores encontraron que la incidencia de EA relacionados con la asistencia en urgencias y detectados durante la hospitalización fue de 0,76\% (IC95\%: 0,51\%-1,1\%). La proporción de EA que se produjeron en el área de urgencias fue de un $3 \%$ (IC: 1,6\%-4,5\%) del total de EA. El 55,2\% de los EA fueron leves, el $31 \%$ moderados y el $13,8 \%$ graves. El 48,3\% de los EA se relacionaron con los cuidados, el $20,7 \%$ con la medicación, el $13,8 \%$ con las infecciones nosocomiales, el $6,9 \%$ se asociaron a algún procedimiento y el 10,3\% al diagnóstico. El 44,8\% de los EA tuvieron como consecuencia un incremento de la estancia, y en un $24,1 \%$ el EA condicionó el ingreso y, por lo tanto, toda la hospitalización fue debida a éste. Esta carga suponía como mediana 3 días en los EA que alargaron la estancia y de 5 días en los que causaron un reingreso. El 75,9\% de los EA fueron evitables, aunque no se encontró relación entre la evitabilidad y la gravedad. Para los autores, la edad es un factor de vulnerabilidad del paciente, ya que los mayores de 65 tienen el doble de EA que los menores de 65 años. Este hallazgo es resaltable puesto que estudios sobre la inadecuación de ingresos señalan al colectivo mayor de 65 como aquel de mayor riesgo de admisión inadecuada ${ }^{1}$. También los factores de riesgo intrínsecos y extrínsecos influyen, ya que los pacientes con factores de riesgo intrínsecos tienen casi tres veces más EA que aquellos que no los presentan, y los que tiene factores de riesgo extrínsecos tiene más del doble de EA que aquellos que no los presentan.

Así pues, y según los datos extraídos del estudio ENEAS, los EA que se originan en urgencias constituyen una proporción no despreciable del total de los EA debidos a la propia asistencia sanitaria. Los EA originados en urgencias son, con mayor frecuencia, leves, están relacionados con los cuidados y contienen, a su vez, una elevada proporción de evitables. Todas estas cualidades que permiten caracterizar los EA de urgencias como específicos, justifican la necesidad de la adecuada identificación de los mismos para la elaboración de estrategias que minimicen su aparición. Además, la sobreinstrumentalización de los cuidados en urgencias incrementa el riesgo de los pacientes.

\section{Estudio APEAS}

Los datos del estudio APEAS (estudio sobre los efectos adversos asociados a la atención primaria) ${ }^{11}$, si bien no son extrapolables a la situación en los SU, sí pueden servir, en cierto grado, como un parámetro de medición de la repercusión que la aparición de EA puede causar sobre los mismos. En algunos casos los resultados pueden ser también aplicables, especialmente en la valoración de facto- 
Tabla 2. Tipos de eventos adversos detectados en estudio ENEAS.

\begin{tabular}{|c|c|}
\hline Tipos de EA & $\%$ \\
\hline Relacionados con los cuidados & $7,63 \%$ \\
\hline Úlcera por presión & 3,66 \\
\hline Quemaduras, erosiones y contusiones (incluyendo fracturas consecuentes) & 2,90 \\
\hline EAP e insuficiencia respiratoria & 0,61 \\
\hline Otras consecuencias de la inmovilización prolongada & 0,46 \\
\hline Relacionados con la medicación & $37,4 \%$ \\
\hline Nauseas, vómitos o diarrea secundarios a medicación & 4,89 \\
\hline Prurito, rash o lesiones dérmicas reactivas a fármacos o apósitos & 4,89 \\
\hline Otros efectos secundarios de fármacos & 4,43 \\
\hline Mal control de la glucemia & 2,90 \\
\hline Hemorragia por anticoagulación & 2,75 \\
\hline Agravamiento de la función renal & 1,98 \\
\hline Hemorragia digestiva alta & 1,98 \\
\hline Retraso en el tratamiento & 1,68 \\
\hline Insuficiencia cardiaca y shock & 1,53 \\
\hline IAM, AVC, TEP & 1,37 \\
\hline Neutropenia & 1,37 \\
\hline Alteraciones neurológicas por fármacos & 1,37 \\
\hline Alteración del ritmo cardíaco o actividad eléctrica por fármacos & 1,37 \\
\hline Hipotensión por fármacos & 1,07 \\
\hline Infección oportunista por tratamiento inmunosupresor & 0,92 \\
\hline Desequilibrio de electrolitos & 0,92 \\
\hline Cefalea por fármacos & 0,76 \\
\hline Tratamiento médico ineficaz & 0,76 \\
\hline Reacciones adversas a agentes anestésicos & 0,46 \\
\hline Relacionados con infección nosocomial & $25,34 \%$ \\
\hline Infección de herida quirúrgica & 7,63 \\
\hline ITU nosocomial & 6,87 \\
\hline Otro tipo de infección nosocomial o infección nosocomial sin especificar & 3,36 \\
\hline Sepsis y shock séptico & 2,90 \\
\hline Neumonía nosocomial & 2,60 \\
\hline Bacteriemia asociada a dispositivo & 1,98 \\
\hline Relacionados con un procedimiento & $25,04 \%$ \\
\hline Hemorragia o hematoma relacionadas con intervención quirúrgica o procedimiento & 9,31 \\
\hline Lesión en un órgano durante un procedimiento & 3,05 \\
\hline Otras complicaciones tras intervención quirúrgica o procedimiento & 2,14 \\
\hline Intervención quirúrgica ineficaz o incompleta & 1,68 \\
\hline Desgarro uterino & 1,37 \\
\hline Neumotórax & 1,07 \\
\hline Suspensión de la intervención quirúrgica & 0,92 \\
\hline Retención urinaria & 0,92 \\
\hline Eventración o evisceración & 0,92 \\
\hline Deshicencia de suturas & 0,76 \\
\hline Hematuria & 0,76 \\
\hline Complicaciones locales por radioterapia & 0,61 \\
\hline Seroma & 0,76 \\
\hline Adherencias y alteraciones funcionales tras intervención quirúrgica & 0,46 \\
\hline Complicaciones neonatales por parto & 0,31 \\
\hline Relacionados con el diagnóstico & $\mathbf{2 , 7 5 \%}$ \\
\hline Retraso en el diagnóstico & 1,53 \\
\hline Error diagnóstico & 1,22 \\
\hline Otros EA & $1,83 \%$ \\
\hline
\end{tabular}

Tomado y adaptado de Ministerio de Sanidad y Consumo. Estudio nacional sobre los efectos adversos ligados a la hospitalización. Madrid: Ministerio de Sanidad y Consumo; 2006, sobre un total de 665 Eventos adversos detectados. 
res de riesgo, al tratarse al fin y al cabo la atención urgente una atención sobre pacientes de la comunidad. El estudio APEAS cifra aproximadamente una tasa de EA del $18,63 / 1000$ ciudadanos atentidos en atención primaria. Un $57,4 \%$ de los pacientes que sufren un EA tienen algún factor de riesgo intrínseco, siendo la hipertensión arterial, hiperlipemia, diabetes, obesidad, depresión e insuficiencia cardíaca aquellos concurrentes con mayor frecuencia. Es fácil observar, en estos casos, que la medicación juega un papel importante en todos ellos. Un $24,8 \%$ de los EA que apa- recen en $\mathrm{AP}$ generan una visita a un SU o atención especializada, dando lugar a un ingreso en un 5,8\% de los casos.

Una valoración interesante que se extrae en el estudio APEAS y que consideramos puede ser aplicable a los SU, es el análisis de causas que pueden motivar un EA (Fig. 1). Los factores relacionados con la medicación se convierten en los más frecuentes, pero vale la pena una llamada de atención sobre aquellos relacionados con los problemas de comunicación (25\%) entre profesionales y/o con el enfermo, situaciones también muy habituales en los SU.

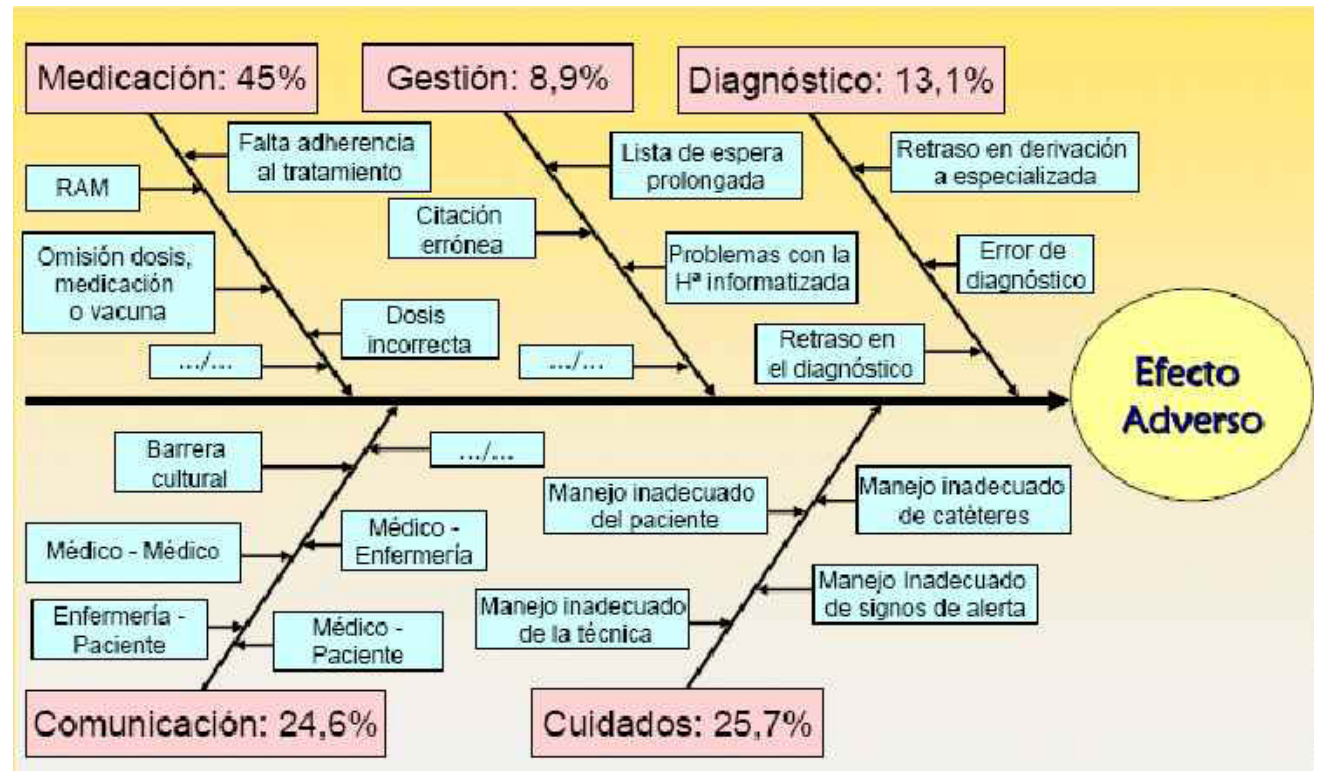

Fuente: Estudio APEAS. J Aranaz y col ${ }^{11}$

Figura 1. Análisis de causas que pueden motivar un E.A.

\section{Estudio EVADUR}

El estudio EVADUR (eventos adversos en servicios de urgencias) desarrollado por la Sociedad Española de Medicina de Urgencias y Emergencias (SEMES) cuya característica principal es la recogida de datos prospectiva, con seguimiento de los pacientes durante su estancia en urgencias y posteriormente hasta 7 días después del alta. Se trata de un proyecto ambicioso, que cuenta con la participación de 25 hospitales españoles y que se encuentra todavía abierto en fase de estudio. Los datos preliminares (datos no publicados), obtenidos del pilotaje del estudio, cifran la incidencia de eventos adversos en un $13,8 \%$, de los cuales un $9 \%$ corresponderían a EA que llegan al paciente, mientras que un $4,8 \%$ serían incidentes que se detectan antes de 
llegar al mismo, pero no por ello despreciable por el riesgo que pudieran provocar algún EA. A pesar de que estos datos deben interpretarse con cautela (se trata de una primera valoración del estudio...) y que el estudio sigue aún vigente, son cifras que nos acercan más a la situación en nuestros SU. Varios datos a valorar de estos resultados preliminares: más de la mitad de los EA detectados (57\%) se produjeron durante la atención de urgencias médicas; en un 49\% de los casos, se consideró que el EA podía haber sido evitado; los enfermos con nivel de gravedad 1 ó 2 del SET (o sistema de triaje similar) tuvieron un mayor riesgo de sufrir algún tipo de EA; los efectos debidos a la medicación empleada, la repetición de procedimientos y/o el empeoramiento del estado del paciente fueron las consecuencias clínicas más habituales, cuyos principales factores causales fueron los problemas de comunicación entre profesionales $\mathrm{y} /$ con el paciente, los relacionados con la medicación (dosis incorrecta, omisiones, reacciones adversas a medicamentos) y los relacionados con los cuidados (manejo inadecuado de la técnica, paciente y/o cateter). A destacar que sólo en un $20 \%$ de los casos se registró en la historia clínica la aparición del EA o incidente (lo cual confirma la baja sensibilidad de los estudios retrospectivos) y en uno de cada tres EA detectados aparecieron posteriormente al alta (EA tardíos).

\section{ESTRATEGIAS PARA LA PRÁCTICA CLÍNICA SEGURA EN LOS SERVICIOS DE URGENCIAS}

La gestión de la seguridad del paciente (SP) en urgencias comprende la identificación y priorización de riesgos, el diseño y aplicación de planes de mejora y la evaluación del resultado de su implantación. Cuando se empieza a trabajar en este ámbito es probable que no se disponga todavía de datos y resultados propios (o de entornos cercanos) o que éstos sean escasos. En todo caso, siempre son de gran ayuda las aportaciones concretas que ofrecen diversas organizaciones significativas en el cam- po de la SP para priorizar las actuaciones de análisis y mejora en una institución en particular.

Estos organismos, ya sea de modo individual o en forma de alianzas entre ellos, establecen políticas que proporcionan recomendaciones establecidas en base a los EA o incidentes comunicados y de estudios realizados. Asimismo, aportan el análisis causal y la valoración de su repercusión por los mismos notificantes o por un equipo de expertos, el diseño de planes de acción y los resultados de su aplicación así como herramientas metodológicas adaptables a nuestro entorno que nos facilitan el trabajo.

De su revisión podemos establecer un conjunto de las políticas prioritarias que hacen referencia a las siguientes áreas y destacan aquellos aspectos de las mismas relacionados con la atención urgente:

1. Seguridad relacionada con la medicación

2. Seguridad relacionada con la infección

3. Seguridad relacionada con la identificación

4. Seguridad relacionada con la comunicación/información

5. Seguridad relacionada con la prevención de riesgos específicos

6. Promoción de una cultura de seguridad

\section{Seguridad relacionada con la medicación en urgencias}

Los SU son un cruce natural de caminos entre los distintos niveles de atención sanitaria, hecho que los convierte en un observatorio ideal de todas las bondades y contradicciones de nuestro sistema sanitario, así como en un punto donde las decisiones terapéuticas tomadas tienen gran impacto más allá del propio ámbito, tanto en atención primaria, si el paciente es dado de alta, como en atención hospitalaria, si el paciente es hospitalizado. Por los motivos que ya se ha comentado anteriormente (Tabla 1), los SU se convierten en el área 
del hospital que genera un mayor número de problemas evitables relacionados con la medicación.

Se han publicado varios estudios sobre problemas relacionados con la medicación (PRM) que son causa de consulta médica a un SU, tanto en nuestro medio como en otros países. Las duplicidades de tratamientos equivalentes o las omisiones de tratamientos domiciliarios relevantes, las dosificaciones inadecuadas a la condición del paciente son algunos de los PRM que se han identificado en estos estudios. Tuneu y $\mathrm{col}^{12}$ observaron que un 19\% de las urgencias hospitalarias eran causadas por PRM y los más frecuentes fueron la indicación no tratada y las reacciones adversas. Climente y $\mathrm{col}^{13}$ analizaron la prevalencia, característica y factores de riesgo asociados a los PRM que requirieron ingreso hospitalario y estimaron el impacto económico en 2.300 estancias/año y 370.000 euros al año. Baena y col $^{14}$ presentaron los resultados de un trabajo sobre urgencias hospitalarias, encontrando un prevalencias de PRM como motivo de consulta en el $33,2 \%$ de los ingresos, con una evitabilidad del $73,6 \%$ y un coste asociado de 12 millones de euros/ año. Zed y col, en un estudio sobre 1.017 visitas, identificaron a 122 visitas como debidas a PRM (12\%), de las que un $9,8 \%$ se consideraron graves ${ }^{15}$. El estudio ENEAS ha mostrado que un $4 \%$ de los pacientes tuvieron un EA relacionado con la medicación y ésta fue la primera causa $(37,4 \%)$ de todos los EA recogidos, así como la de los que ocasionan ingreso hospitalario $(29,8 \%)^{9}$.

Sin embargo, un elevado porcentaje de PRM se consideran prevenibles y las distintas intervenciones que se han realizado en diversos entornos han conllevado mejoras reales en seguridad clínica ${ }^{16}$. Las actuaciones en el ámbito de la seguridad relacionada con la medicación en los SU se centran actualmente en tres áreas: utilización de medicamentos, seguridad con los medicamentos de alto riesgo y conciliación de la medicación. Algunos SU han incorporado la figura de un farmacéutico clínico a tiempo parcial o completo, cuya actuación está dirigida específicamente a la reducción de los PRM en dichas tres áreas ${ }^{17}$.

\section{El sistema de utilización de medicamentos}

El sistema de utilización de medicamentos es un proceso cada vez más complejo que engloba subprocesos de selección, prescripción, validación, dispensación, administración y seguimiento ${ }^{18}$. Esta complejidad (unida a otros factores como la cantidad de medicación que se prescribe diariamente, las decisiones clínicas e interconexiones asociadas a cada componente, los múltiples profesionales que intervienen, etc.) conlleva un riesgo mayor de que se produzcan $\mathrm{PRM}^{19}$. A nivel de los $\mathrm{SU}$ los datos recogidos en el período de 1999 a 2003 (casi 11.000 errores notificados) por el programa MedMARX SM desarrollado por la United States Pharmacopeia (USP) mostraron que un $45 \%$ pertenecían a la administración (casi un tercio atribuibles a dosis inapropiada) y el $29 \%$ a la prescripción, mientras que de los que habían provocado daño al paciente, un $12 \%$ se atribuían a la monitorización y el $6,7 \%$ a la administración ${ }^{20}$. Las políticas de mejora a este respecto son múltiples. Puede mencionarse, a modo de consulta, las recomendaciones efectuadas por el Ministerio de Sanidad y Consumo a raíz de un informe de evaluación de seguridad de los medicamentos a nivel hosptialario Evaluación de la seguridad de los sistemas de utilización de medicamentos en los hospitales españoles (2007). Informe mayo 2008(http://www. msc.es/organizacion/sns/planCalidadSNS/ docs/evaluacionSeguridadSistemasMedicamentos.pdf).

\section{Seguridad en medicación de alto riesgo}

Se incluye en esta categoría aquella medicación que presenta una alta probabilidad de producir EA o incluso pueden provocar la muerte cuando se realiza un uso indebido ${ }^{21}$. Dentro de este grupo, y que afectan a los SU, se menciona la insulina, los opiáceos, las sales de potasio endovenosas, el cloruro sódico a concentraciones mayores de $0,9 \%$ y la heparina (por extensión, terapia anticoagulante en general) ${ }^{22}$. Las soluciones concentradas de electroli- 
tos son objeto de recomendaciones y alertas específicas por parte de distintas organizaciones. Cabe realizar también acciones para mejorar la seguridad de los medicamentos de estrecho intervalo terapéutico, que se definen como aquéllos en los que la dosis que logra el efecto deseado está muy próxima a la que causa efecto tóxico (citostáticos, agonistas adrenérgicos, hipoglucemiantes orales, digoxina, litio, etc.). El documento "Prácticas para mejorar la seguridad de los medicamentos de alto riesgo", editado por el Ministerio de Sanidad y Consumo $^{23}$, es un estudio completo sobre el tema con recomendaciones sobre acciones de mejora.

\section{Conciliación de la medicación}

Los llamados problemas derivados de la conciliación de la medicación (es decir, las discrepancias no intencionadas que se producen entre los medicamentos que el paciente tomaba antes del ingreso y los prescritos durante su ingreso en el SU, hospital o en el momento del alta) es un fenómeno que constituye una de las principales causas de $\mathrm{PRM}^{24}$. Estudios realizados en paises anglosajones han puesto de manifiesto que el problema es relevante: el porcentaje de pacientes con problemas derivados de la conciliación varía de un 26,9 a un $65 \%^{25,26}$, algunos de los cuales originaron efectos adversos en el paciente e incluso ingresos hospitalarios desde el $\mathrm{SU}^{24-26}$. La omisión de medicación parece ser el PRM de conciliación más habitual en los $\mathrm{SU}^{27}$.

Ante esta situación, se ha sugerido la puesta en marcha de acciones de mejora dirigidas a disminuir los PRM en los SU, mediante la incorporación de un farmacéutico clínico en los mismos ${ }^{24}$. La implantación de programas de conciliación de medicación ha comportado una reducción de EM en un $85 \%^{27,28}$. La mayoría de organismos competentes en seguridad clínica incluyen la conciliación dentro de sus estrategias. En EEUU la conciliación es obligatoria para que la JCAHO acredite a un centro sanitario y establece conciliacio- nes modificadas o simplificadas para servicios o procesos en que la prescripción de medicación es mínima o por muy corto tiempo (urgencias, cirugía ambulatoria entre otros $)^{29}$.

\section{Seguridad relacionada con la infección}

Aproximadamente un $8,7 \%$ de los pacientes hospitalizados presentan infecciones nosocomiales según la $\mathrm{OMS}^{30}$. En nuestro país el estudio EPINE ${ }^{31}$ del año 2007 otuvo una prevalencia en torno al $7 \%$. El estudio ENEAS ${ }^{9}$ halló que el $25,3 \%$ de los EA detectados estaban relacionados con la infección nosocomial (la segunda causa más frecuente después del uso de medicamentos) y que ésta era la responsable de un 19,9\% de los EA que precisaron de reingreso hospitalario. En este mismo estudio, un 13,8\% de los EA generados en el SU fueron infecciones nosocomiales y un $75 \%$ eran previsiblemente evitables, que contrasta con el $56 \%$ de la evitabilidad del resto de hospitalización. La medida clave para minimizar el riesgo de infecciones es el lavado de manos, mediante la realización de talleres que impliquen a todo el personal del SU, siguiendo las recomendaciones de la OMS (http://www.who.int/patientsafety/en/)

\section{Seguridad relacionada con la comunicación/información}

La información y su comunicación suelen ser factores subyacentes de muchos de los EA de los apartados anteriores. No obstante, hay datos suficientes que permiten considerarla como un grupo aparte. La JCAHO documentó en un estudio del año 2003 que las anomalías en la comunicación eran las responsables de cerca de un $60 \%$ de errores médicos de los cuales un $75 \%$ acabaron en muerte ${ }^{32}$. Asimismo, la comunicación era la primera causa raíz de los eventos centinela recogidos por la JCAHO en el año 2005. La mayoría de gestores de riesgos de los países occidentales concuerdan en que hasta el 
$80 \%$ de las reclamaciones por mala praxis son atribuibles a fallos en la comunicación o falta de habilidades comunicativas, generalmente atribuibles a los médicos ${ }^{32}$. Las recomendaciones suelen hacer referencia a puntos críticos que afectan a los SU, tales como:

- Comunicación de la información durante las transferencias del paciente entre los diferentes pasos de la continuidad asistencial, ya sea dentro del mismo SU (cambio de turno, de servicio, traslados, consultas en turno de noche, etc.) como entre los distintos servicios y/o organizaciones diferentes (alta, traslado interhospitalario, etc. $)^{1}$.

- Promoción de sistemas seguros, claros y estandarizados de registro y transmisión de información (especialmente la no presencial) y de confirmación de determinado tipo de la misma (resultados críticos, etc.) así como estandarización de las abreviaturas, acrónimos, símbolos y medidas.

- Comunicación al paciente cuando se ha producido un EA en el mismo. Las conductas de evitación, de justificaciones poco claras y/o de barreras de cualquier tipo, conducen inevitablemente a reclamaciones y denuncias, situaciones gravosas tanto para el profesional como para el paciente y familiares. Los esfuerzos dirigidos a mejorar este aspecto de comunicación son todavía asignaturas pendientes en el mundo sanitario y en los SU.

- Asimismo, la Ley 41/2002 de autonomía del paciente y de derechos y obligaciones en materia de información y documentación clínica hace referencia a que "la comprensión del riesgo es básica para estar en disposición de tormar decisiones". La información debe ser adecuada y conveniente al proceso y la situación concreta frente al término de "completa" que alude la Ley General de Sanidad.

\section{Seguridad relacionada con identificación (SI)}

La identificación incorrecta de pacientes conduce a PRM de transfusión, de pruebas complementarias, de procedimientos a personas incorrectas y de alta de bebés a familias equivocadas ${ }^{33}$. La estrategia de mejorar la identificación a través de pulseras identificativas (PI) es la más difundida. La NPSA recoge las recomendaciones sobre cómo han de ser empleadas las $\mathrm{PI}^{34}$. Sin embargo, la implantación de SI mediante PI en los SU es muy deficitaria. Un estudio realizado en $75 \mathrm{SU}$ de nuestro medio y publicado en el año 2008 encontraba que un $41 \%$ de los SU hospitalarios no utilizaban sistema de identificación alguno de los enfermos, cifra que aumentaba al $49 \%$ en caso de urgencias pediátricas. Estos datos contrastaban con el resto de datos declarados por los centros: un 90,7\% declaraban utilizar algún sistema de identificación de los pacientes; un $85 \%$ utilizaban PI, y estaba protocolizado sólo en el 67,2\% de los centros $^{35}$. Existe, por tanto, una discrepancia entre lo declarado en hospitalización y lo que se hace en los SU. Aproximadamente, un $7 \%$ de los EA que acontecen en los SU podrían ser causados por problemas de identificación de pacientes, según datos provisionales del estudio EVADUR (datos no publicados). Todos estos resultados muestran que, en los SU, la identificación es una asignatura pendiente. Las estrategias dirigidas al incremento de implantación de PI desde los SU pueden reducir el riesgo de EA por dicho problema.

\section{Seguridad relacionada con la prevención de riesgos específicos}

En este apartado se incluyen la prevención de EA relacionados con los cuidados, con los procedimientos, con la valoración general y el diagnóstico, no incluidos en apartados anteriores. Sin pretender ser exhaustivos. Se destacan aquéllos con mayor relevancia para SU:

- La identificación de determinados riesgos en los pacientes atendidos que 
conducen a una morbimortalidad significativa así como la adopción de medidas de prevención son otro grupo de políticas con gran número de recomendaciones. Entre ellas destacamos las establecidas por el documento "Prácticas seguras simples: recomendadas por agentes gubernamentales para la prevención de efectos adversos (EA) en los pacientes atendidos en hospitales" editado por el Ministerio de Sanidad y Política Social ${ }^{16}$. Asimismo existen recomendaciones orientadas a que las instituciones identifiquen los riesgos inherentes a los pacientes que atienden.

- La adopción de sistemas de trabajo capaces de identificar síntomas y/o signos de alarma previos a que la condición del paciente evolucione a un estado crítico y la instauración precoz de medidas adecuadas reducen la morbimortalidad. En los SU, la implantación de los sistemas de triaje ha sido el principal avance en los últimos años ${ }^{36}$. También, en un plano más específico, la implantación de guías de actuación para atención de patologías de alto impacto (código ictus, código IAM, código sepsis) en los SU ha reducido la morbimortalidad de las mismas al reducir las demoras diagnósticas y terapéuticas que pueden producirse en el proceso asistencial desde el propio $\mathrm{SU}^{37-39}$.

- También es objeto de recomendación específica la adopción de precauciones en el manejo de conexiones de catéteres y tubos así como en las inmovilizaciones de pacientes. En este apartado se pueden incluir la seguridad en técnicas específicas como sondajes, intervenciones quirúrgicas, inyección epidural, etc. Aunque se pueda incluir en el apartado de administración de medicación, deben mencionarse aquí las recomendaciones sobre la administración de medicamentos por via parenteral ${ }^{40}$.

- Otro grupo de recomendaciones generales hace referencia a la adecua- ción estructural (física y humana) del $\mathrm{SU}$ a las necesidades de asistencia que debe prestar (por ejemplo, dotación de profesionales sanitarios), su organización interna, las guías y protocolos mínimos que deben existir y conocer, así como los sistemas de evaluación de resultados, como elementos para garantizar una política de mejora continua de la seguridad del paciente ${ }^{41}$.

- Finalmente, las recomendaciones sobre la identificación de riesgos (mapa de riesgos) de los procesos de atención urgente, como medida preventiva que permita la puesta en marcha de acciones de mejora en la reducción de los mismos. En este sentido, existen actualmente grupos de trabajo desarrollando un análisis modal de fallos y efectos (AMFE) sobre dichos procesos en $\operatorname{los} \mathrm{SU}^{42}$.

\section{Promoción de una cultura de seguridad y creación de un equipo y liderazgo}

Dos puntos básicos para que la progresión en SP sea efectiva son construir una cultura de seguridad (CS) y construir y liderar un equipo de personas motivadas. Ello precisa de una atmósfera de confianza mutua en que todos los miembros del servicio puedan hablar libremente sobre problemas de seguridad y sus posibles soluciones sin el temor a estigmas o represalias, con la garantía de que los efectos adversos se valoran de modo imparcial y justo y que se realizarán acciones para la mejora. La promoción de la CS es una recomendación general que acompaña a todas las que se realizan ante alertas o incidentes concretos que hemos mencionado ${ }^{43}$. El clima de CS en los SU debe iniciarse mediante sesiones informativas por estamentos y favorecer la participación del personal interesado. Debe pasarse del enfoque centrado en la persona al enfoque sistémico basado en el sistema, tal como se recoge en la tabla 3 . 
Tabla 3. Cultura de seguridad: desde el enfoque centrado en la persona hacia el enfoque sistémico centrado en el sistema.

\begin{tabular}{|l|l|}
\hline \multicolumn{1}{|c|}{ De: } & \multicolumn{1}{c|}{ A: } \\
\hline La seguridad como propiedad accesoria & La seguridad como componente esencial \\
\hline Conducta reactiva & Actitud proactiva \\
\hline Autoritarismo & Trabajo en equipo e intercambio de ideas \\
\hline Individualismo y actitud artesanal & Trabajo en equipo y estandarización de prácticas \\
\hline Ocultación y silencio & Comunicación abierta y transparencia \\
\hline Atención centrada en el profesional & Atención orientada al paciente \\
\hline Focalizada en el profesional que se equivoca & Centrada en el sistema que favorece el error \\
\hline $\begin{array}{l}\text { Búsqueda del culpable } \\
\text { ¿Qué? ¿Quién? ¿Cuándo? ¿Cómo? }\end{array}$ & $\begin{array}{l}\text { Análisis de las causas. } \\
\text { ¿Qué?¿Cuándo?¿Cómo? ¿Por qué? } \\
\text { ¿Qué hacer para que no se repita? }\end{array}$ \\
\hline Hacer «todo lo posible». & Hacer «todo lo adecuado» \\
\hline
\end{tabular}

Es interesante conocer cuál es la CS de la que partimos ("baseline assesment»), para poder valorar aquellos puntos cuya mejora conviene priorizar y valorar los progresos que se irán consiguiendo. A este fin disponemos de herramientas. Destacan entre otras, la encuesta de seguridad de la $\mathrm{AHRQ}^{44}$. Esta agencia esponsorizó el desarrollo de un cuestionario que permite evaluar la CS del paciente tanto a nivel del hospital como de servicios o unidades específicas, cuestionario que ha sido validado en España por el Ministerio de Sanidad.

(http://www.msc.es/organizacion/sns/ planCalidadSNS/docs/Analisis_cultura_SP_ ambito_hospitalario.pdf)

En ese sentido, en el informe de la AHRQ correspondiente al año $2008^{45}$, realizado sobre un total de 160.176 cuestionarios recogidos en 519 hospitales, se analizaron, entre otros, las impresiones de cerca de 8.000 profesionales de servicios de urgencias y emergencias que valoraron el grado de seguridad del servicio como excelente en un $16 \%$ y muy buena en un $46 \%$ de los encues- tados Por el contrario, «sólo» un 45\% manifestó no haber comunicado ningún evento adverso en los últimos 12 meses, mientras que en el grupo general fue del 52\%) $\mathrm{Al}$ igual que en el grupo general, la dimensión mejor valorada fue la de equipo de trabajo en la unidad (79\% respuestas positivas) y la peor valorada la de respuesta no punitiva al error (38\% respuestas positivas, datos inferiores al del grupo general que era del 44\%). En nuestro país la SEMES ha administrado la encuesta en 729 profesionales de SU de 9 comunidades autónomas (datos pendientes de publicar), en donde se constata que el trabajo en equipo en la unidad es la dimensión mejor valorada (67\% respuestas positivas) y las peor valoradas son la dimensión de recursos humanos (31\% respuesta positivas) y el apoyo de las direcciones o gerencia (21\% respuestas positivas). La puntuación de la seguridad del paciente en los SU obtuvo un valor medio de 6,4 puntos. Estos resultados deberían ayudar a orientar la política de implantación y mejora de la CS en las organizaciones de los SU. 


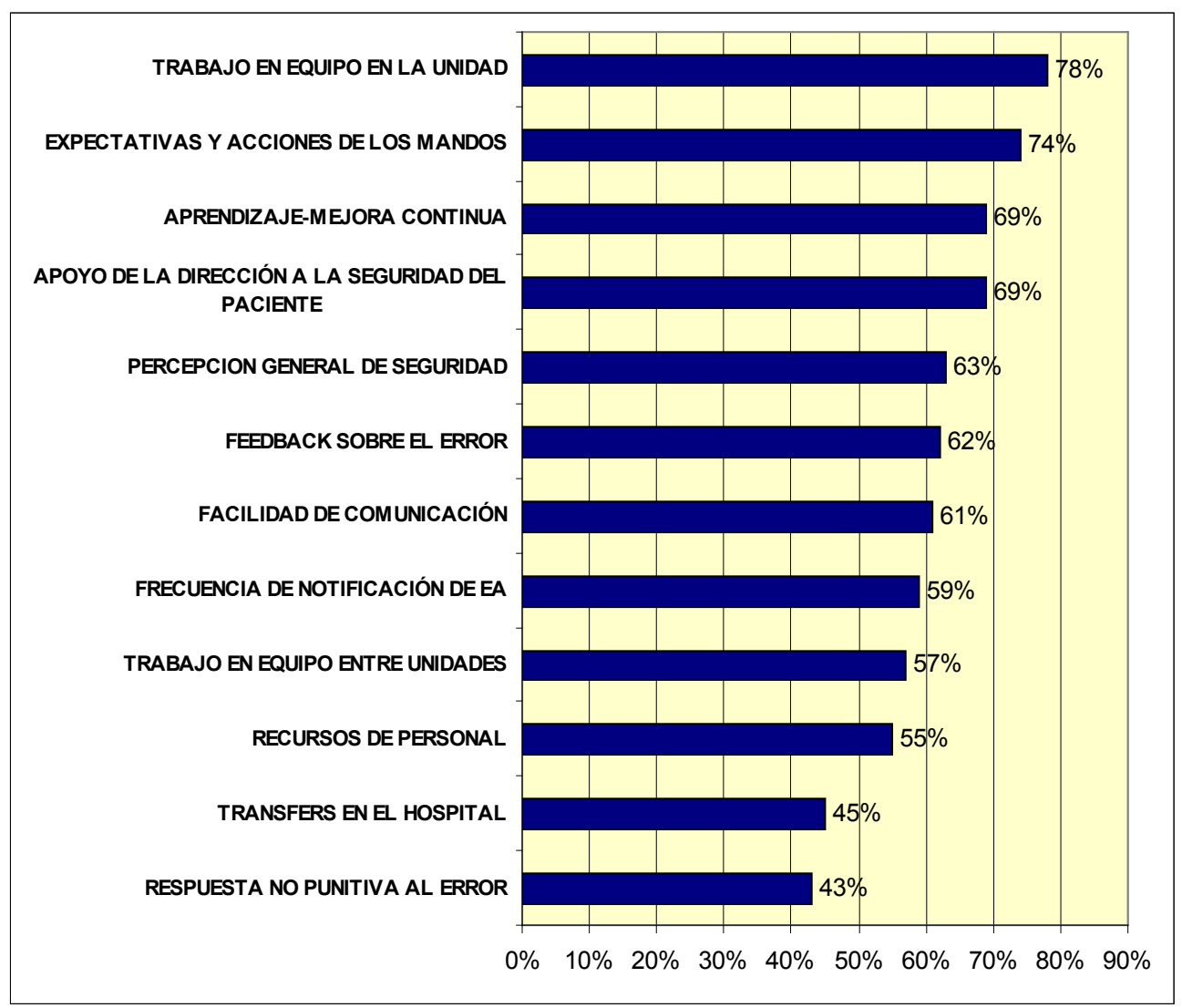

En marco azul, áreas positivas. En marco rojo, área de mejora. Las puntuaciones por debajo de $50 \%$ se consideran negativas.

Figura 2. Encuesta AHRQ (informe 2008, EEUU). Resultados globales agrupados por áreas.

\section{INDICADORES DE SEGURIDAD DEL PACIENTE EN URGENCIAS}

La construcción y validación de indicadores en SP es un campo de trabajo en el cual se han abocado las sociedades científicas, instituciones y la administración. En este sentido son destacables:

\section{Los "triggers"}

Los «triggers" son criterios que nos permiten identificar posibles eventos adversos relacionados con la medicación. Representan signos, síntomas o situaciones que ponen sobre la pista de la probable existencia de un efecto adverso. Es una herramienta que se ha mostrado efectiva para medir el nivel de daño que puede provocar la medicación en una organización sanitaria. La forma de utilización más común es la retrospectiva, útil para detectar eventos adversos y para proponer estrategias para mejorar en la gestión del medicamento. Cuando se encuentran, hay que proceder a una evaluación más detallada para ver si efectivamente se ha producido un efecto adverso ${ }^{46}$. Han sido desarrollados por el Institute of Health Improvement de Estados Unidos, y actualmente existen seis herramientas de este tipo para diversos entornos y tipos de efecto adverso (medicamentos, intervención quirúrgica, unidad de cuidados intensivos (UCI), UCI neonatal 
y consultas externas). Algunos grupos intentan validar estas herramientas en SU, aunque no se dispone aún de resultados

\section{Los indicadores centinela}

Son aquéllos en los que un solo caso ya es indicativo de problema y debe conducir a un análisis de causas para rediseñar el proceso afectado, de forma que no vuelva a producirse el evento detectado. Ejemplo de sucesos centinela son los que la JCAHO utiliza como casos en los que debe seguir obligatoriamente un análisis de causas raíz ("Root Cause Analisis") y los "Never Events" o "Eventos que no deben ocurrir nunca" del Nacional Quality Forum. (National Quality Forum. Serious Reportable Events in Health Care-2006 Update. Wahington: The National Quality Forum; 2007).

\section{Indicadores de sociedades científicas}

Las sociedades científicas de medicina de urgencias han publicado manuales sobre indicadores de calidad en los servicios de urgencias, recogiendo aspectos de seguridad y riesgo. Así, de los 103 indicadores de la Societat Catalana de Medicina $d^{\prime}$ Urgència (SCMU) ${ }^{47}$ elaborados en el año 2001 y utilizados por la (SEMES) como base de sus próximas recomendaciones sobre indicadores, un 54\% hacían referencia a aspectos relacionados con la seguridad del paciente, como lo hacían el 51\% de los 89 indicadores de la Sociedad Española de Urgencias Pediátricas ${ }^{11}$ y el $75 \%$ de los 24 indicadores propuestos por la Asociación Española de Toxicología (2006) ${ }^{12}$. En general estos indicadores son tanto de resultado como de proceso y de estructura y se basan en desarrollos originales así como en adaptaciones de la bibliografía y en la experiencia internacional.

El Ministerio de Sanidad y Consumo ha publicado recientemente el manual «Construcción y validación de indicadores de buenas prácticas sobre seguridad del paciente ${ }^{48}$. Gran parte de estas "buenas prácticas" tienen su inicio y/o aplicación desde el SU.

\section{GESTIÓN DE RIESGOS EN UN SU: HERRAMIENTAS PARA EL ANÁLISIS DE LA SEGURIDAD DEL PACIENTE}

La gestión de riesgos tiene una metodología bien establecida y existen distintos modelos que se pueden aplicar a cualquier sector de actividad. Tal como ya se ha comentado anteriormente, un programa de gestión de riesgos aplicado a la prevención de EA en un SU ha de incluir la identificación y priorización de riesgos, el diseño y aplicación de planes de mejora y la evaluación del resultado de su implantación.

El análisis de la seguridad lo podemos hacer de una manera proactiva, es decir, detectar los riesgos y prevenir, o de manera reactiva, analiza las causas de un evento ya acontecido con el objetivo de poner en marcha acciones para reducir el riesgo de nueva aparición. Disponemos de diferentes herramientas que pueden aplicarse en un programa de gestión de riesgos destinados a la mejora de la SP en un servicio clínico. Es necesario, sin embargo, la formación y especialización de profesionales en su uso para la obtención de resultados aplicando una correcta metodología. En este sentido, la SEMES desarrolla periódicamente actividades formativas con el objetivo de especializar a los profesionales de urgencias en el manejo de estas herramientas. En la tabla 4 se sugiere, a modo de propuesta, la utilización de herramientas de análisis que pueden ser útiles en los SU. Entre ellas, queremos destacar, por la facilidad de implantación, las ventajas de los sistemas de registro y notificación, tanto de EA como de incidentes ya que a través de ellos podemos:

- Identificar los puntos débiles del sistema y analizar sus causas

- Aumentar la conciencia de los problemas de seguridad y facilitar el compromiso de los profesionales

- Identificar barreras que previenen de situaciones adversas que puedan desencadenar episodios graves

- Detectar y comunicar las incidencias permite aproximarse a una previ- 
sión de eventos adversos posibles y refuerza la necesidad de puesta en marcha de acciones de mejora proactivas.
- Monitorizar incidentes añade más valor a la mejora de la calidad, ya que se abordan los problemas de seguridad antes de que ocurran.

Tabla 4. Herramientas recomendadas para el análisis de riesgos en un servicio de urgencias

\begin{tabular}{|l|}
\hline $\begin{array}{c}\text { Cómo puedo saber la cultura /clima sobre seguridad en mi servicio de urgencias? } \\
\text { Mediante la encuesta de cultura seguridad (AHRQ) }\end{array}$ \\
\hline $\begin{array}{c}\text { Cómo puedo saber si los procedimientos en mi Servicio de Urgencias son seguros? } \\
\text { - Conocer los procesos ("contexto»): análisis de los procesos del servicio } \\
\text { - Indicadores / eventos centinelas/ triggers }\end{array}$ \\
\hline $\begin{array}{c}\text { ¿Cómo puedo conocer los eventos que ocurren? } \\
\text { A través de fomentar las notificaciones de EA }\end{array}$ \\
\hline $\begin{array}{c}\text { ¿Cómo debo analizar los eventos detectados? } \\
\text { Mediante análisis causal (ACR/London) }\end{array}$ \\
\hline $\begin{array}{l}\text { ¿Cómo puedo prevenir? } \\
\text { Mediante análisis modal de fallos y efectos (AAMFE) de los procesos de atención urgente }\end{array}$ \\
\hline
\end{tabular}

Fuente: Tomás Vecina S, Roqueta Egea F, Chanovas Borras M Seguridad del Paciente como objetivo estratégico ¿de qué estamos hablando?. Monografias Emergencias 2009, 5: 1-8.

El principal inconveniente de los sistemas de registro y notificación es que no consiguen la inclusión de la mayor parte de los incidentes y EA. Un estudio reciente ${ }^{49}$ que compara los EA obtenidos mediante estudio retrospectivo de historias clínicas y registros en el sistema de notificación voluntario detecta que sobre el total de EA detectados el $93,5 \%$ se obtiene de la revisión retrospectiva de historias, un $16,6 \%$ mediante el sistema de notificación, un $10,2 \%$ se detectan con ambos métodos y un $6,5 \%$ tan solo se detectan mediante la notificación por no estar documentado en la historia clínica. Es evidente la infranotificación en estos sistemas pero también pone de manifiesto su utilidad ya que permite detectar EA que no se detectarían en la revisión sistemática de historias clínicas. Entre las barreras citadas para el desarrollo del proceso de notificación, a juicio de la Agencia Nacional para la Seguridad del Paciente británica, el temor a ser culpado y el temor a riesgos médico-legales son dos de los principales factores explicativos de la falta de notificación en muchos $\operatorname{casos}^{50}$. Por ello, es necesario que en los sistemas de notificación no se emplee información identificativa del paciente o personal y se garantice la confidencialidad y el carácter anónimo de los datos.

\section{Agradecimientos}

Los autores quieren agradecer a los Dres. Femí Roqueta Egea y Manel Chanovas Borrás su inestimable ayuda y colaboración científica en la elaboración de este manuscrito.

\section{BIBLIOGRAFÍA}

1. Chanovas Borras M, Campodarve I, Tomas Vecina S. Eventos adversos en los servicios de urgnecias: ¿El servicio de urgencias como sinónimo de inseguridad clínica para el paciente? Monografías Emergencias 2007; 3: 7-13.

2. Tomás S, Duaso E, Ferrer JM, Rodríguez M, PorTA R, Epelde F. Evaluación del uso apropiado de un área de observación de urgencias mediante el Appropriateness Evaluation Protocol: un análisis de 4.700 casos. An Med Intern (Madrid) 2000; 17: 229-237.

3. Peiro S, Meneu R, Roselló ML, Portella E, CarBONELL-SANChís R, FeRnÁndez C et al. Validez del protocolo de evaluación del uso inapropiado 
de la hospitalización. Med Clin (Barc) 1996; 107: 124-129.

4. Gómez JiménEz J. Urgencia, gravedad y complejidad: un constructo teórico de la urgencia basado en el triaje estructurado. Emergencias 2006; 18: 156-164.

5. López Andujar L, Aluama Alcántara M, PérezMontaut Merino I, Cartelle Pérez T, Casado Martínez JL, Tomás Vecina S. Acreditación de Servicios de Urgencias y Emergencias. Todo Hospital 2003; 198: 456-461.

6. Declaración de profesionales por la seguridad del paciente. Ministerio de Sanidad y Consumo 2006. http://www.msc.es/organizacion/sns/planCalidadSNS/pdf/excelencia/ opsc_sp4.pdf (acceso noviembre 2009)

7. Forster AJ, Rose GWN, van Walraven C, Stiell I. Adverse event following and emergency department visit. Qual Saf Health Care 2007; 16: 17-22.

8. De Vries EN, Ramrattan MA, Smorenburg SM, Gouma DJ, Boermeester MA. The incidence and nature of in-hospital adverse events: a systematic review. Qual Saf Health Care 2008; 17: 216-223.

9. Estudio nacional sobre los efectos adversos ligados a la hospitalización. ENEAS 2005 Informe. Febrero 2006 Ministerio de Sanidad y Consumo.

10. Requena Puche J, Aranaz Andes JM, Gea VelázQuez de Castro MT, Miralles Bueno JJ, Limón Ramírez R, Rey TALEns M et al. Efectos adversos asociados a la asistencia sanitaria en los servicios de urgencias hospitalarios. Mapfre Medicina 2007; 18 (Supl. 2): 89-97.

11. Estudio sobre la seguridad de los pacientes en atención primaria de salud. APEAS. Ministerio de Sanidad y Consumo.

12. Tuneu L, García-Peláez M, López S, Serra G, Alba $\mathrm{G}$, DE IRAIA $\mathrm{C}$ et al. Problemas relacionados con los medicamentos en pacientes que visitan un servicio de urgencias. Pharm Care Esp 2000; 2: 177-192.

13. Climente M, Quintana L, Martínez G, Atienza A, JiMÉnEz N. Prevalencia y características de la morbilidad relacionada con los medicamentos como causa de ingreso hospitalario. Aten Farm 2001; 3: 9-22.

14. Baena M, Calleja M, Romero J, Vargas J, Jiménez J, FAus M. Validación de un cuestionario para la identificación de problemas relacioandso con los medicamentos en usuarios de un servicio de urgencias hospitalario. Ars Pharm 2001; 42 : 147-171.
15. Zed PJ, Abu-Laban RB, Balen RM Loewen PS, HoHl CM, BRUBACHER JR et al. Incidence, severity and preentability of medication-related visits to the emergency department: a prospective study. CMEAJ 2008; 178: 1563-1569.

16. Prácticas para mejorar la seguridad de los medicamentos de alto riesgo. Ministerio de Sanidad y Consumo (acceso septiembre 2008). http://www.msc.es/organizacion/sns/ planCalidadSNS/ec03_doc.htm

17. Carter M, Allin D, Scott A, Grauer D. Pharmacist-acquired medication histories in a university hospital emergency department. Am J Health Sys Pharm 2006; 60: 2500-2503.

18. A call to action: eliminate handwritten prescriptions within 3 years. electronic prescribing can reduce medication errors. Huntingdon Valley, PA: ISMP 2000. http://www.ismp. org/newsletters/acutecare/articles/whitepaper.asp?ptr=y (Acceso septiembre 2008)

19. Otero MJ, Martín RM, Robles MD, Codina C. Errores de medicación». Farmacia Hospitalaria. 3a Edición. SEFH. 2002, 713-747.

20. Santell JP, Hicks RW, Cousins DD. Medication errors in emergency department settings - 5 year review. The United States Pharmacopeia, Rockville, Maryland Center for the Advancement of patient Safety, poster presentado en junio de 2004 en el American Society of Health-Systems Pharmacists Summer Meeting (Las Vegas, Nevada). http://www. usp.org/hqi/patientSafety/resources/posters/posterEmergencyDept5yr.html (Acceso septiembre 2008)

21. Cohen MR, Smetzer JL, Tuohy NR, KILo CM. High-alert medications: safeguarding against errors. En: Cohen MR, editor, Medication Errors. 2nd ed. Washington (DC): American Pharmaceutical Association, 2007 317-341.

22. Relación actualizada a 2007 de medicamentos de alto riesgo ISMP (acceso septiembre 2008). http://www.ismp-espana.org/ficheros/ medicamentos_alto_riesgo.pdf

23. Prácticas para mejorar la seguridad de los medicamentos de alto riesgo, Ministerio de Sanidad y Consumo (acceso septiembre 2008). http://www.msc.es/organizacion/sns/ planCalidadSNS/ec03_doc.htm

24. Delgado O, Anoz L, Serrano A, Pico JN. Conciliación de la medicación. Med Clin (Barc) 2007; 129: 343-348.

25. Cornish PL, Knowles SR, Marchesano R, Tam $\mathrm{V}$, Shadowitz S, JuURLINK DN et al. Unintended medication discrepancies at the time of hos- 
pital admission. Arch Intern Med 2005; 165 : 424-429.

26. Gleason KM, Groszek JM, Sullivan C, Rooney D, BARNARD C, Noskin GA. Reconciliation of discrepancies in medication histories and admission orders of newly hospitalized patients. Am J Health Syst Pharm. 2004; 61: 1689-1695.

27. Juanes A. Conciliación de la medicación domiciliaria en los SUH. Experiencia CSPT. Jornada de conciliación de la medicación. Experiencias en hospitales, Societat Catalana Farmacología Clínica, Barcelona 3 junio 2008 (acceso septiembre 2008). http://www.academia.cat/societats/farmcl/jornades/altres/ conciliacio08/docs/annaj.pps

28. Massachusetts Coalition for the Prevention of Medical Error http://www.macoalition. org/initiatives.shtml\#2 (acceso septiembre 2008).

29. The Joint Commission, Hospital Accreditation Program 2009 Chapter: National Patient Safety Goals http://www.jointcommission. org/GeneralPublic/NPSG/09_npsgs.htm

30. Mayon-White RT, Duce G, Kereselidze T, TikomiRov E. An international survey of the prevalence of hospital acquired infection. J Hosp Infect 1988; 11 (Suppl A): 43-48.

31. Prevalencia de las infecciones en los hospitales españoles. Estudio EPINE. Resultados de los estudios de 2004, 2005, 2006 y 2007 y evolución 1990-2007: 18 años. www.sempsph.com/sempsph/attachments/135_nota_ web_EPINE1990-2007.pdf (acceso septiembre 2008 .

32. Woods MS. How communication complicates the patient safety movement, Patient Safety \& Quality Healthcare, may/june 2006 www. psqh.com/mayjun06/dun.html (acceso septiembre 2008).

33. WHO/JC. Solución 2. Identificación de pacientes. http://www.ccforpatientsafety.org/ fpdf/presskit/PS-Solution2.pdf (acceso septiembre 2008).

34. Sevdalis N, Norris B, Ranger C, Bothwell S. Designing evidence-based patient safety interventions: the case of the UK's National Health Service hospital wristbands. J Eval Clin Pract 2009; 15: 316-322.

35. Martínez L, Gimeno Victoria, Anglés R, Bañeres Torralba J, Manzanera R. Sistemas de identificación de pacientes en hospitales de Cataluña. Rev Calidad Asistencial 2008; 23: 158-163.

36. Castro Delgado R, Arcos González P, Rodríguez SOLER A. Sistema sanitario y triaje ante una pandemia de gripe: un enfoque desde la salud publica. Emergencias 2009; 376-381.

37. Gómez-Angelats E, Bragulat E, Obach V, GómezChoco M, SÁnchez M, Miró O. Resultados alcanzados con la puesta en marcha del circuito "Codigo ictus" en un gran hospital. Emergencias 2009; 21: 105-113.

38. STEAd LG. El protocolo "código ictus": una llamada de acción. Emergencias 2009; 21: 85-86.

39. Aguirre Tejedo A, Echarte Pazos JL, Mínguez Masó S, Supervía Caparrós A, Skaf Peters E, CamPODARVE Botet I. Implementación de un "código sepsis grave» en un servicio de urgencias. Emergencias 2009; 21: 255-261.

40. Llodrà V, Puigventós F, Barroso MA, Martínez A, Comas F, Giné J et al. Impacto de una guía de administraciñon de medicamentos por vía parenteral en las unidades de enfermería. Farm Hosp 1996; 20: 245-249.

41. Florido Mancheño Jl, Capilla Roncero M. Revisión de los estándares de acreditación para los servicios de urgencias de hospitales: a propósito de las jornadas de 48 horas semanales. Emergencias 2009; 21: 301-305.

42. Orrego Villagran C. Análisis de la seguridad clínica y herramientas de evaluación proactiva. Villagran Orrego C. Monografía Emergencias 2007; 3: 18-21.

43. "La seguridad del paciente en 7 pasos" Agencia de Calidad del Sistema Nacional de Salud, Ministerio de Sanidad y Consumo, noviembre de 2005. Traducción y resumen de "Seven Steps to patient Safety", Nacional Patient Safety Agency del National Healt Service de Reino Unido.

44. Sorra JS, Nieva VF. Hospital Survey onb Patient Safety Culture. (Prepared by Westat, under Contract No. 290-96-0004). AHRQ Publication No. 04-0041. Rockville, MD: Agency for Healthcare Research and Quality. Septiembre 2004.

45. Sorra J, Famolaro T, Dyer N, Nelson D, Khanna K. Hospital Survey on Patient Safety Culture 2008 Comparative Database Report (prepared by Westat, Rockville, MD, under contract № 233-02-0087, Task Order 18). AHRQ Publication № 08-0039. Rockville, MD: Agency for Healthcare Research and Quality. March 2008.

46. Introduction to trigger tools for identifying adverse events, institute for healthcare improvement. http://www.ihi.org/IHI/Topics/ PatientSafety/SafetyGeneral/Tools/IntrotoTriggerToolsforIdentifyingAEs.htm (último acceso 14 Enero 2008). 
47. Felisart J, Requena J, Roqueta F, Saura RM, Suñol R, TomÀs S. Serveis d’Urgències: indicadors per mesurar els criteris de qualitat de l'atenció sanitària. Barcelona: Agencia d’Avaluació de Tecnologia i Recerca Mèdiques. Servei Català de la Salut. Departament de Sanitat i Seguretat Social. Generalitat de Catalunya. Junio 2001. Disponible en: http:// www.gencat.net/salut/depsan/units/aatrm/ pdf/br0101es.pdf

48. Construcción y validación de indicadores de buenas prácticas sobre seguridad del paciente. Madrid: Ministerio de Sanidad y Consumo 2008.
49. Baba-Akbari Sari A, Sheldon TA, Cracknell A y Turnbull A. Sensitivity of routine system for reporting patient safety incidents in an NHS hospital: retrospective patient case note review. BMJ [revista electrónica] 2007; 334; 79 (consultado 08-12-2008). Disponible en: http://bmj.com/cgi/content/full/334/7584/79

50. Osborn S, Williams S. Seven steps to patient safety. An overview guide for NHS staff. Londres: The National Patient Safety Agency 2a Edicion, Abril 2004. (consultado 08-12-2008). Disponible en: http://www.npsa.nhs.uk/nrls/ improvingpatientsafety/patient-safety-toolsand-guidance/7steps/ 